\title{
The Use of Gaze in Human and Machine Vision
}

\author{
Dana Ballard \\ University of Texas at Austin, USA \\ dana@cs.utexas.edu
}

\begin{abstract}
The modern study of human vision increasingly emphasizes its function in natural behaviors and complex computer games. The more complex settings call for visually directed multitasking management, so that a venue almost absent a decade ago is now a central concern. With that development has seen the study of gaze allocation. This study has been accelerated with the development of inexpensive eye trackers that are robust for moving subjects in both natural and virtual environments. Yet another trend has been the use of models that are programmed with learning algorithms. A final trend is that as these models become ever more sophisticated, they are engendering two-way interactions of the techniques between human and robotic approaches. The presentation will describe examples of all these developments.
\end{abstract}

